\title{
Effective microscopic theory of quantum dot superlattice solar cells
}

\author{
U. Aeberhard
}

Received: 30 September 2011 / Accepted: 15 December 2011 / Published online: 25 December 2011

(C) The Author(s) 2011. This article is published with open access at Springerlink.com

\begin{abstract}
We introduce a quantum dot orbital tight-binding non-equilibrium Green's function approach for the simulation of novel solar cell devices where both absorption and conduction are mediated by quantum dot states. By the use of basis states localized on the quantum dots, the computational real space mesh of the Green's function is coarse-grained from atomic resolution to the quantum dot spacing, which enables the simulation of extended devices consisting of many quantum dot layers.
\end{abstract}

Keywords Quantum dot · Solar cell · NEGF

\section{Introduction}

Extended quantum dot superlattices (QDSL) as found in third generation solar cell devices (Green 2000; Martí et al. 2006) consist of a large number of weakly coupled quantum dots, with a number of involved atoms exceeding the limit of what may still be handled by even today's largest supercomputers. For that reason, atomistic (Kirchartz et al. 2009) and even microscopic continuum (Jiang and Green 2006) simulations of QDSL absorbers make use of the symmetry and periodicity properties present in the idealized structure. However, in real devices, the finite number of periods, large built-in fields and any kind of disorder make the electronic structure deviate considerably from the minibands found for the latter and hence disqualify such simplifications. Furthermore, even for narrow electronic minibands and gaps in the phonon spectrum, the charge carriers will be subject to various kinds of scattering, causing dissipation and preventing purely coherent transport in the device. For the determination of the device characteristics it is thus desirable to derive a computational model that on the one hand can still be handled numerically by making use of the localized nature of the QD wave functions, and which on the other hand is able to cope with all of the above mentioned deviations from the idealized structure, thanks to being based on a quantum-kinetic theory of

U. Aeberhard $(\bowtie)$

IEK-5 Photovoltaik, Forschungszentrum Jülich, 52425 Jülich, Germany

e-mail: u.aeberhard@fz-juelich.de 
photogeneration, recombination and transport, including injection and extraction of carriers at contacts, similar to the non-equilibrium Green's function (NEGF) approach developed for quantum well solar cells in Aeberhard and Morf (2008), Aeberhard (2011a,b). The present paper introduces such a model and applies it to the investigation of (photo)carrier localization and extraction in selectively contacted silicon quantum dot absorber arrays under varying internal field conditions.

\section{Theoretical approach}

The NEGF theory that forms the core of the model provides a quantum version of the Boltzmann transport equation for the charge carriers (Kadanoff and Baym 1962; Keldysh 1965; Datta 1995; Binder and Koch 1995), which includes tunneling transport and non-local rate terms for scattering, generation and recombination, which are all essential processes in the photovoltaic regime of device operation. For the representation of the electronic system, the present hybridization approach uses the perturbative expansion of the QDSL wave function in terms of the eigenstates of the isolated dots. The resulting molecular orbital approach can be interpreted as a tight-binding (TB) theory with quantum dot orbitals replacing the atomic orbitals, and is in spirit similar to the first NEGF models of quantum well superlattices for quantum cascade laser simulations (Wacker 2002; Lee and Wacker 2002) and to NEGF models of polaron transport in QD superlattices (Vukmirovic et al. 2007). The orbitals $m=1,2, \ldots, M$ of QD $i=1,2, \ldots, N_{D}$ with energies $\varepsilon_{i m}$ are used to represent the field operators for single particle quantum dot states, $\hat{\Psi}(\mathbf{r}, t)=\sum_{i, n} \psi_{i n}(\mathbf{r}) \hat{d}_{i n}(t)$, where $\hat{d}_{i n}$ annihilates an electron in state $|n\rangle$ on the QD at position $\mathbf{R}_{i}$. The non-interacting nearest-neighbor tight-binding Hamiltonian in this QD orbital basis is

$$
\mathcal{H}_{0}(t)=\sum_{\langle i, j\rangle} \sum_{m, n=1}^{M} t_{i m, j n} \hat{d}_{i m}^{\dagger}(t) \hat{d}_{j n}(t)+\sum_{i=1}^{N_{D}} \sum_{m=1}^{M} \tilde{\varepsilon}_{i m} \hat{n}_{i m}(t),
$$

where $\langle i, j\rangle$ are nearest-neighbor sites, $\mathbf{t}$ is the hopping matrix, $\hat{n}$ the density operator and $\tilde{\varepsilon}_{i m}=\varepsilon_{i m}+\bar{U}_{i}$, with $\bar{U}_{i}$ the average value for the Hartree potential of Coulomb interaction at the dot position. The heterostructure potential does no longer appear explicitly, since it has been considered in the determination of the TB-parameters $\boldsymbol{\varepsilon}$ and $\mathbf{t}$,

$$
t_{i m, j n}=-\int d^{3} r \psi_{i m}^{*}(\mathbf{r}) \Delta U(\mathbf{r}) \psi_{j n}(\mathbf{r})
$$

with $\Delta U(\mathbf{r})=H_{S L}(\mathbf{r})-\sum_{\mathbf{R}_{i}} H_{Q D}\left(\mathbf{r}-\mathbf{R}_{i}\right)$, where $H_{S L}$ is the superlattice Hamiltonian and $H_{Q D}$ is the Hamiltonian of the isolated QD. The Hamiltonian (1) is used in the equations for the steady state non-equilibrium Green's functions for the device states, which read

$$
\begin{aligned}
& \mathbf{G}^{R}(E)=\left[\left\{\mathbf{G}_{0}^{R}(E)\right\}^{-1}-\Sigma^{R I}(E)-\Sigma^{R C}(E)\right]^{-1}, \\
& \mathbf{G}^{\lessgtr}(E)=\mathbf{G}^{R}(E)\left[\Sigma^{\lessgtr I}(E)+\Sigma^{\lessgtr C}(E)\right] \mathbf{G}^{A}(E), \\
& \mathbf{G}_{0}^{R}(E)=\left[(E+i \eta) \mathbb{1}-\mathbf{H}_{0}\right]^{-1}, \quad \mathbf{G}^{A}(E)=\left[\mathbf{G}^{R}(E)\right]^{\dagger},
\end{aligned}
$$

where $\mathbf{G} \equiv\left[G_{i m, j n}\right]$ with $G_{i m, j n}(E)=\int d \tau e^{i E \tau / \hbar} G_{i m, j n}(\tau), \quad \tau=t^{\prime}-t$, and $G_{i m, j n}\left(t, t^{\prime}\right)=-\frac{i}{\hbar}\left\langle\hat{T}_{\mathcal{C}}\left\{\hat{d}_{i m}(t) \hat{d}_{j n}^{\dagger}\left(t^{\prime}\right)\right\}\right\rangle$ the quantum-statistical non-equilibrium average defined on the Keldysh contour $\mathcal{C}$ (Keldysh 1965). In the above equations, the self-energy terms $\Sigma^{\alpha I}(\alpha \in\{\lessgtr, R, A\})$ describe the renormalization of the single charge carrier Green's 
function due to the interactions relevant for the photovoltaic device operation, i.e., coupling to photons, phonons and other charge carriers, and are obtained within many-body perturbation theory on the level of the self-consistent first Born approximation. As these self-energies are functionals of the carrier Green's functions, they need to be computed self-consistently with the latter. The additional self-energy $\Sigma^{\alpha C}$ encodes the coupling of the QD states to the states of the contacts, which in this case are assumed as bulk electrodes. Details on the general derivation and explicit form of the self-energies can be found in Aeberhard (2011b).

Once the NEGF have been determined, they immediately provide any physical observable on the single particle level. For instance, the density of states is obtained from the NEGF and the QD eigenfunctions via

$$
\mathscr{D}(\mathbf{r}, E)=\sum_{i, j} \sum_{m, n} A_{i m, j n}(E) \psi_{i m}^{*}(\mathbf{r}) \psi_{j n}(\mathbf{r}),
$$

where $\mathbf{A} \equiv i\left(\mathbf{G}^{R}-\mathbf{G}^{A}\right)$ is the charge carrier spectral function. In terms of the tight-binding interdot hopping and Green's function elements, the current flowing between two quantum dots can be expressed as ${ }^{1}$

$$
J_{i}=\frac{2 e}{\hbar} \int d E \operatorname{tr}\left\{\mathbf{t}_{i i+1} \mathbf{G}_{i+1 i}^{<}(E)-\mathbf{G}_{i i+1}^{<}(E) \mathbf{t}_{i+1 i}\right\} .
$$

\section{Implementation for $\mathrm{Si}-\mathrm{SiO}_{2}-\mathrm{SiC} \mathrm{QD}$ heterostructure absorbers}

Since the wave functions and energies of the quantum dot eigenstates are determined separately for each individual dot, the corresponding computational domain is small enough to allow, in principle, for the use of accurate ab-initio methods. In this paper, however, since the main focus is on the general formalism, the orbitals are approximated via the superposition of three separate 1D solutions for a simple one-band effective mass model. In order to agree with full real space calculations, the local variation of the electrostatic mean-field potential in principle needs to be included in the solution of the Schrödinger equation, and to be computed self-consistently with the carrier density via Poisson's equation. In this first approach, a linear potential drop resulting in a constant field is assumed and the orbitals are computed only once for the flat-band case. This means that quantum-confined Stark effects resulting from the distortion of the orbitals due to the local field are not considered at the present stage.

The structure under investigation is shown schematically in Fig. 1a. It consists of decoupled arrays of 20 cubic silicon quantum dots of $3 \mathrm{~nm}$ side length embedded laterally in $\mathrm{SiO}_{2}$ and vertically separated by thin SiC layers of $1 \mathrm{~nm}$ thickness. ${ }^{2}$ The QD array is coupled to contacts on both ends, and the regions adjacent to the contacts are doped to provide a built-in field. The non-selfconsistent band diagram is shown in Fig. 1b. The internal field depends on the built-in potential $V_{b i}$, determined by the doping, and the bias voltage $V_{b i a s}$ applied at the right contact, the latter corresponding to a finite separation of the chemical potentials associated with the equilibrated electrodes. In order to prevent leakage current flow and to enable charge separation even in absence of an internal electric field, minority carrier contacts are closed artificially, the contacts thus becoming carrier-selective.

At this stage of implementation, the optical modes considered are those of a homogeneous medium and with monochromatic occupation, and only radiative recombination is

\footnotetext{
${ }^{1}$ The trace is over orbital indices.

${ }^{2}$ A similar structure was recently fabricated as a candidate for an all-silicon tandem solar cell component (Ding et al. 2011).
} 
(a)

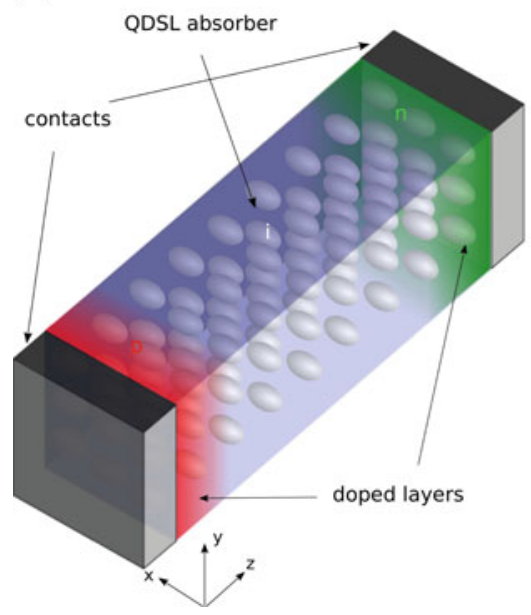

(b)

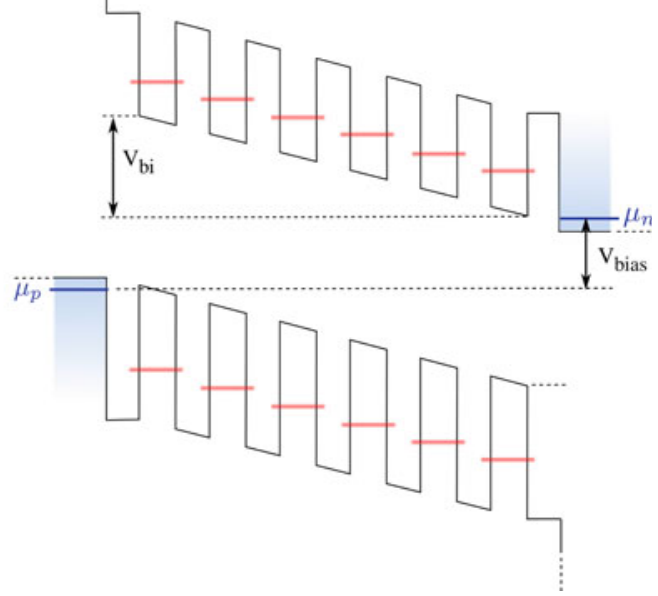

Fig. 1 (Color online) a Schematic structure and $\mathbf{b}$ resulting band diagram of a $p-i-n$-QD-array absorber solar cell device. The resulting net potential drop $V_{b i}$ in the absorbing region is determined by the combination of the built-in potential due to doping and the terminal bias voltage $V_{\text {bias }}$, the latter corresponding to the separation of the chemical potentials $\mu_{n, p}$ at the contacts

considered. Dissipation of energy is modelled via coupling to a single optical (bulk) phonon mode described within the deformation potential approximation. The energy of the optical branch was chosen at $E_{\text {phon }}=0.06 \mathrm{eV}$. Additional broadening of the electronic states is obtained from elastic scattering with acoustic phonons, using again the appropriate bulk deformation potentials.

\section{Numerical results}

Figure 2 shows the local as well as the spatially integrated density of states (LDOS and DOS) of the selectively contacted QD-array structure from Fig. 1 for vanishing internal field $\left(V_{b i}=V_{\text {bias }}=0\right)$, in the case where only the lowest QD orbital is considered. Due to the finite number of dots, the density of states shows discrete maxima, but as a consequence of the flat band situation, the states are completely delocalized over the structure, such that on every QD site, the multiplicity of states amounts to the number of QDs, as can be recognized in the magnification of the shaded region (Fig. 3). The difference between electrons and holes is due to the differing values for effective masses and barrier height. While the weak mutual coupling of QD states results in a very narrow "miniband" in the active device region, the LDOS is strongly broadened close to the carrier selective contacts, which is due to the efficient coupling to the broad-band bulk electrode DOS. This hybridization with bulk electrode states is also reflected in the integrated DOS.

In the flat band situation, extraction of photogenerated charge carriers is efficient, since all the absorbing states are directly connected to the contact states. However, the situation changes drastically in the presence of a finite field, as displayed in Fig. 4. The width of the "miniband" increases in the integrated DOS, but in the LDOS, the number of states per QD with significant spectral weight is reduced, since even for a field as weak as $25 \mathrm{kV} / \mathrm{cm}$, which is close to the values encountered in real devices, the states are spatially localized on a few 


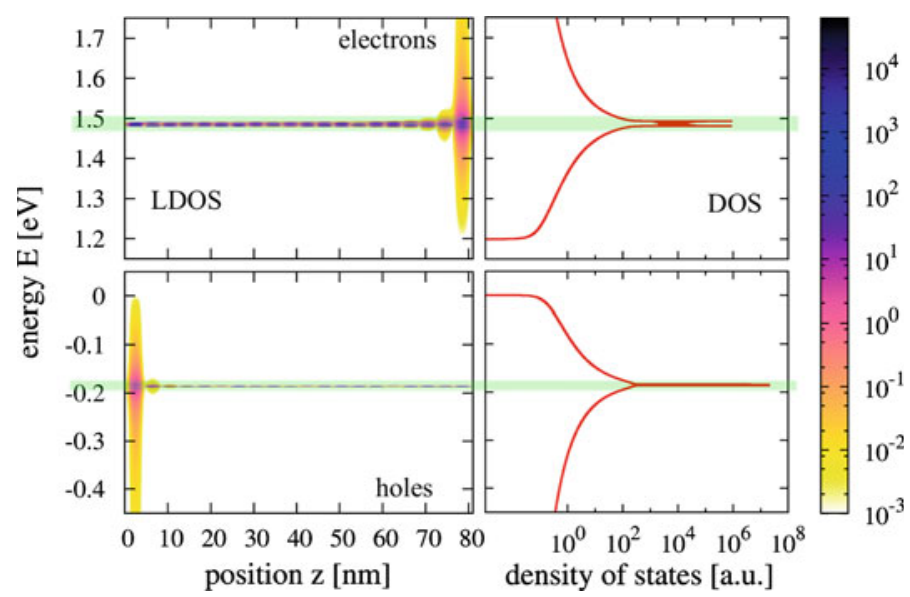

Fig. 2 (Color online) Local density of states (LDOS) and integrated DOS of the selectively contacted QDarray structure from Fig. 1 for vanishing internal field ( $V_{b i}=0$, flat "miniband"). Only the contribution from the lowest orbital is shown. While the weak coupling of the QDs results in a very narrow band, the LDOS close to the carrier selective contacts shows a characteristic broadening due to hybridization with the states of the bulk electrode. The magnification of the shaded area is shown in Fig. 3

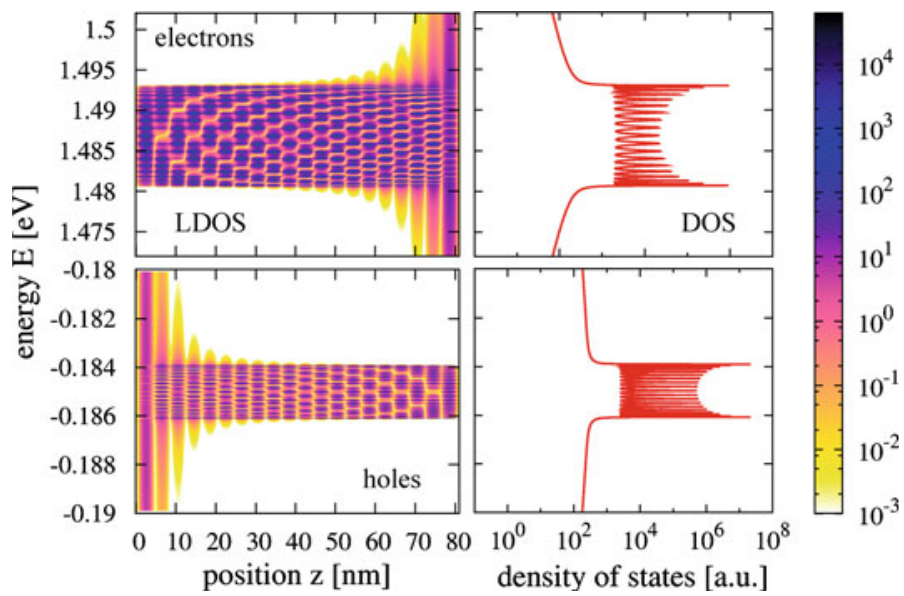

Fig. 3 LDOS and integrated DOS at vanishing built-in field (shaded area from Fig. 2). In this situation, the states are maximally delocalized and extend over entire multi-QD structure. The discrete set of maxima in the LDOS on the QD positions reflect both coupling and finite number of QDs in the structure

QDs. This carrier localization, clearly seen in the magnification shown in Fig. 5, prevents a direct ballistic extraction of photogenerated charge carriers and thus increases the significance of scattering assisted transport ranging from sequential tunneling to hopping between single dots.

The restriction to the lowest orbital leads to an underestimation of the overall inter-dot coupling. In order to study the extraction of excess charge generated under illumination, the single-band hopping matrix element is increased to $0.05 \mathrm{eV}$. To investigate the transport of photogenerated charge carriers under conditions were the states are no longer completely delocalized, i.e., in the situation where inelastic scattering is crucial to enable carrier flow 


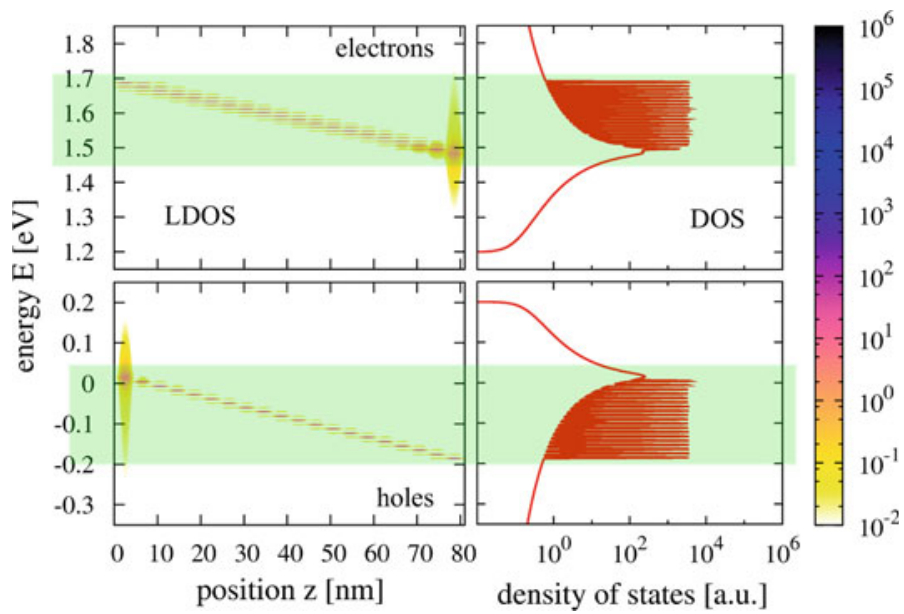

Fig. 4 (Color online) Same as Fig. 2, but for a finite internal field of $25 \mathrm{kV} / \mathrm{cm}$. The field splits the quasiminiband into a broader level structure with reduced local energetic overlap
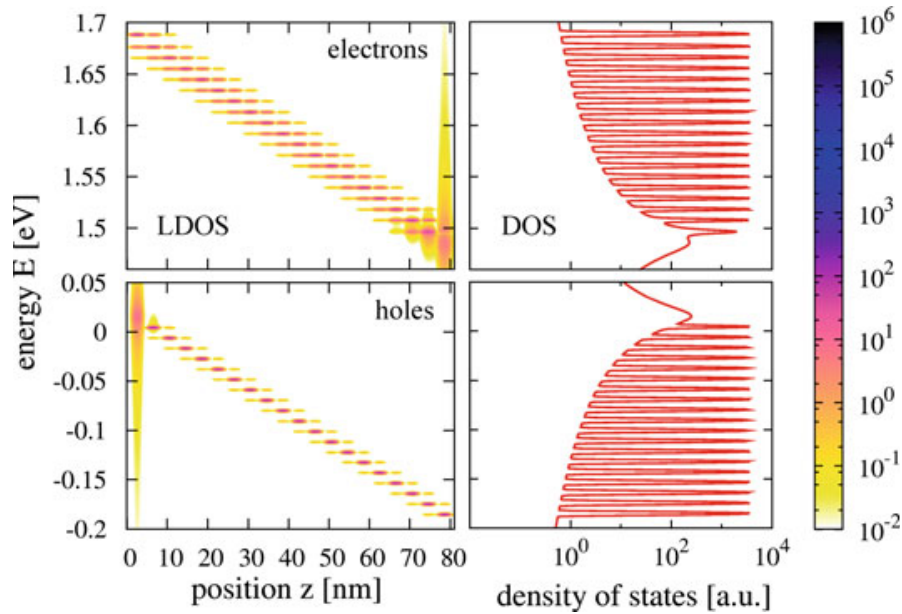

Fig. 5 (Color online) Detailed view of the situation at finite internal field of $25 \mathrm{kV} / \mathrm{cm}$ (shaded area in Fig.4). The field has a strong localizing effect on the QD-array states, which are spatially confined to a small number of neighboring QD. Without the presence of higher states, ballistic transport to the contact is no longer possible in this situation, i.e., energy dissipation via inelastic scattering processes is required to enable photocarrier extraction

between quantum dots, the local photocurrent spectrum is computed for gradually increasing internal field. The result is displayed in Fig. 6 for monochromatic illumination at a photon energy of $1.67 \mathrm{eV}$, corresponding roughly to the effective energy gap. Even though the current spectrum is strongly modified by the internal field, the energy integrated current differs only slightly for different field values, and the overall current, i.e. the sum of electron and hole contributions, is perfectly conserved over the entire device. The strong electron-phonon scattering leads to a fast relaxation within the few QDs over which the states are delocalized, such that photocurrent flow follows the effective band edge. Thus, efficient photocarrier extraction is recovered at the expense of the energy dissipated in the inelastic scattering process. 


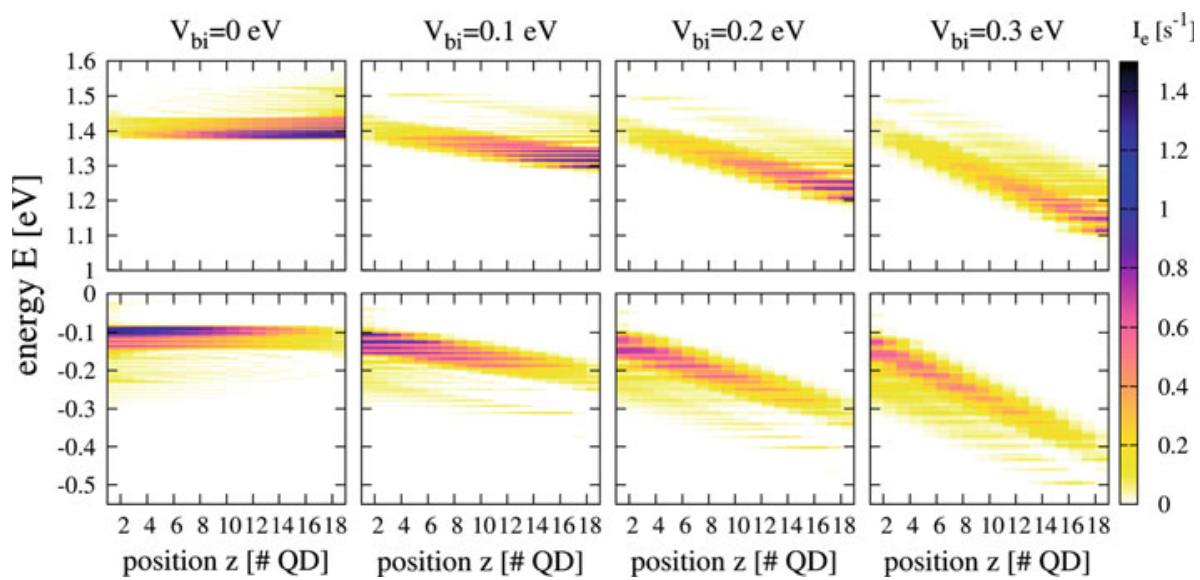

Fig. 6 (Color online) Photocurrent spectrum at photon energy of $1.67 \mathrm{eV}$ for zero applied bias and different values of the built-in field. Due to strong electron-phonon interaction, the photogenerated electron-hole pairs relax to the effective band edge within the delocalization range of a few quantum dot spacings, and photocarrier extraction remains possible even in absence of ballistic current contributions. In spite of the large variation in the current spectrum for different internal fields, the energy integrated current differs only slightly, and the total current (electron + hole) is perfectly conserved over the entire device

\section{Conclusions}

The QDTB-NEGF approach extends the applicability of powerful quantum-kinetic methods to optoelectronic devices based on electronically coupled quantum dot structures, with arbitrary internal fields and spatial arrangement. Application to an array of weakly coupled quantum dot reveals the significant carrier localization for finite electric fields in the absorbing region. For stronger coupling, photogenerated carriers are extracted efficiently also at considerable internal fields via phonon-assisted sequential tunneling and inter-dot hopping.

Acknowledgments Financial support was provided by the German Federal Ministry of Education and Research (BMBF) under Grant No. 03SF0352E.

Open Access This article is distributed under the terms of the Creative Commons Attribution Noncommercial License which permits any noncommercial use, distribution, and reproduction in any medium, provided the original author(s) and source are credited.

\section{References}

Aeberhard, U., Morf, R.: Microscopic nonequilibrium theory of quantum well solar cells. Phys. Rev. B 77, $125343(2008)$

Aeberhard, U.: Theory and simulation of photogeneration and transport in $\mathrm{Si}-\mathrm{SiOx}$ superlattice absorbers. Nanoscale Res. Lett. 6, 242 (2011a)

Aeberhard, U.: Theory and simulation of quantum photovoltaic devices based on the non-equilibrium Green's function formalism. J. Comput. Electron. 10(4), 394-413 (2011b)

Binder, R., Koch, S.W.: Nonequilibrium semiconductor dynamics. Prog. Quantum Electron. 19, 307$462(1995)$

Datta, S.: Electronic Transport in Mesoscopic Systems. Cambridge University Press, Cambridge (1995)

Ding, K., Aeberhard, U., Astakhov, O., Köhler, F., Beyer, W., Finger, F., Carius, R., Rau, U.: Silicon quantum dot formation in SiC/SiOx hetero-superlattices. Energy Procedia 10, 249-254 (2011) 
Green, M.A.: Potential for low dimensional structures in photovoltaics. Mater. Sci. Eng. B 74(1-3), 118$124(2000)$

Jiang, C.W., Green, M.A.: Silicon quantum dot superlattices: modeling of energy bands, densities of states, and mobilities for silicon tandem solar cell applications. J. Appl. Phys. 99, 114902 (2006)

Kadanoff, L.P., Baym, G.: Quantum Statistical Mechanics. Benjamin, Reading (1962)

Keldysh, L.: Diagram technique for nonequilibrium processes. Sov. Phys. JETP 20, 1018-1026 (1965)

Kirchartz T., Seino, K., Wagner, J.M., Rau, U., Bechstedt, F.: Efficiency limits of Si/SiO2 quantum well solar cells from first-principles calculations. J. Appl. Phys. 105, 104511 (2009)

Lee, S.C., Wacker A.: Nonequilibrium Green's function theory for transport and gain properties of quantum cascade structures. Phys. Rev. B 66, 245314 (2002)

Martí, A., López, N., Antolín, E., Cánovas, E., Stanley, C., Farmer, C., Cuadra, L., Luque, A.: Novel semiconductor solar cell structures: the quantum dot intermediate band solar cell. Thin Solid Films 638, 511-512, 638-644 (2006)

Vukmirovic, N., Ikonic, Z., Indjin, D., Harrison, P.: Quantum transport in semiconductor quantum dot superlattices: electron-phonon resonances and polaron effects. Phys. Rev. B 76, 245313 (2007)

Wacker, A.: Semiconductor superlattices: a model system for nonlinear transport. Phys. Rep. 357, 1-111 (2002) 